\title{
LA FORMACIÓN PEDAGÓGICA DEL DOCENTE EN LA FACULTAD DE CIENCIAS DE LA EDUCACIÓN E IDIOMAS DE LA UNIVERSIDAD ESTATAL PENINNSULA DE SANTA ELENA - ECUADOR
}

\author{
THE PEDAGOGIC TRAINING OF THE TEACHERS IN THE FACULTY \\ OF EDUCATION AND LANGUAGES AT STATE UNIVERSITY OF SANTA \\ ELENA PENÍNSULA - ECUADOR
}

\author{
Carlos Cañedo Iglesias, Ph.D. \\ Prometeo SENESCYT - UPSE \\ ccanedo2013@gmail.com. \\ Margarita Lamas González, Ph.D, Docente UPSE \\ Nelly Panchana Rodríguez, MsC. Docente UPSE \\ Yuri Ruiz Rabasco, MsC. Docente UPSE
}

\section{RESUMEN}

El objetivo de este reporte científico consiste en socializar el sistema de formación pedagógica dirigido al perfeccionamiento de la dimensión pedagógica de los profesores que conforman el claustro de la Facultad de Ciencias de la Educación e Idiomas. La contribución teórica radica en la concepción de la teoría y práctica en la preparación pedagógica, atendiendo al contexto, la definición y caracterización de los niveles de formación del claustro de profesores con el fin de lograr el perfeccionamiento de la actividad pedagógica, constituye el aporte teórico ya que en las investigaciones revisadas al respecto no se encontraron evidencias de los objetivos a alcanzar en cada nivel de formación propuesto. En el presente trabajo se demuestra que con la aplicación del sistema de formación pedagógica se contribuye a la formación pedagógica de los docentes implicando el logro de un mayor ingreso y retención de los estudiantes universitarios.

Palabras clave: sistema, formación pedagógica, niveles de formación, ingreso y retención

\begin{abstract}
The objective of this scientific report is to socialize the teacher training system aimed at improving the pedagogical dimension of the teachers who make up the faculty of the Faculty of Education and Language. The theoretical contribution lies in the understanding of the theory and practice in the educational preparation, considering the context, the definition and characterization of the level of training of the teaching staff in order to achieve the improvement of pedagogical activity, is the theoretical contribution and that the revised research about evidence not met the objectives to be achieved each level of training proposed. In the present work it is shown that the application of the system of teacher education contributes to the pedagogical training of teachers involving achieving higher income and retention of college students.
\end{abstract}

Keywords: system, pedagogical training, training stages, entry and retention.

Recibido: mayo 2015 Aprobado: junio 2015

\section{Introducción}

La formación pedagógica del profesorado universitario se considera como uno de los grandes retos de la educación, y constituye la base para la solución de otros grandes problemas planteados a esta. García Ramis L. considera que el autoperfecionamiento docente "es la actividad autotransformadora que presupone el cambio del docente centrado en el dominio y comprensión profunda de los fines y naturaleza de la actuación profesional, incluyendo los mecanismos que facilitan su cambio sistemático y su autoanálisis". Así mismo continúa insistiendo en que "(...) auto perfeccionarse es un constante re-análisis de la información sobre los modos de actuar, los procedimientos, las motivaciones, las conceptualizaciones sobre la labor pedagógica, que generan procesos de búsqueda y transformaciones a partir de la propia experiencia y de la experiencia ajena y que recodifica, reorganiza y sistematiza todo el sistema de trabajo (...) hacia estadios superiores de desarrollo conscientemente determinados"(1). 
Por tal motivo, resulta pertinente dotar a los docentes de las herramientas, vías o mecanismos que desarrollen la capacidad de autoanálisis para su transformación consciente y el tránsito hacia niveles superiores de desarrollo pedagógico. Diversas investigaciones han demostrado la persistencia de una baja actividad meta-cognitiva en los estudiantes, aun en los niveles superiores de la enseñanza lo que está presente también en los propios docentes y se ha demostrado que es causada principalmente por la falta de formación pedagógica del docente en este sentido. Rosenshine y Meister (2) exponen que el buen profesor ha de proporcionar guías, modelos, herramientas para orientar la actividad mental de sus alumnos hacia la consecución de objetivos de aprendizaje, expresando la necesidad de efectuar esta regulación externa para que sea interiorizada y compartida por sus estudiantes.

En opinión de los autores de este reporte, las valoraciones citadas con anterioridad destacan el significado de la formación que deben recibir los profesores como docentes universitarios para su actuación; pero estos fundamentos no reconocen de manera explícita la dedicación a la profesión, que deben tener ellos; rasgo distintivo de la profesión en la Educación Superior, lo que le da sentido a los conocimientos, habilidades, valores y modos de actuación que hay que desarrollar en todos los profesores universitarios mediante la formación postgraduada. Como se aprecia en los párrafos anteriores, los especialistas citados ven la formación del docente desde diferentes paradigmas, pero en común las valoran como parte de la formación permanente y asociada a las exigencias de la formación pedagógica y sus competencias inherentes. La fundamentan en sistemas, modelos y programas que se ofertan en instituciones de Educación Superior en la actualidad; algunos de ellos profundizan en el carácter cada vez más humanista y menos tecnológico que tiene el proceso de formación pedagógica que requiere el profesor universitario para una mejor actuación como docente.

Núñez y Palacio plantean al mismo tiempo los componentes en los que debe realizarse la formación pedagógica del profesor y citan a Álvarez de Zayas; cuando expresa "que el componente académico: contiene el fundamento teórico necesario de los objetivos del programa y básicamente está contenido en las asignaturas, disciplinas o módulos, y carreras donde se desempeña el docente"(3). El componente laboral: abarca toda la actividad práctica a realizar, concebida como un sistema enfocado hacia alcanzar el modo de actuación requerido. Está íntimamente relacionada con su actividad diaria como profesor., es decir, potenciar el acercamiento de la academia a lo laboral e investigativo. Tributa con mucha fuerza a la profesionalización del profesor universitario. El componente investigativo: abarca la actividad del profesor como constructor de los resultados de la investigación realizada, es decir, el profesor capacitado para aplicar los adelantos de la ciencia de la educación.

Dichos componentes se corresponden con las actividades sustantivas de la Educación Superior, donde los docentes tienen un papel importante y constituyen estas actividades de las universidades el escenario donde en la actualidad se realiza la actividad postgraduada, ese es el camino que hoy se está tratando de encausar en las universidades ecuatorianas.

En esa dirección se encuentran diferentes reflexiones en los autores consultados como Addine (4), que señalan la necesidad de articular la teoría con la práctica como condición para el desarrollo de investigaciones que respondan a la solución de necesidades propias de estas ciencias que sean sostenibles en el tiempo, luego que el profesor atraviesa de formas más simples a las más complejas de su formación pedagógica, atendiendo a las necesidades en correspondencia con su nivel de desarrollo profesional.

Los autores de este reporte científico asumen la formación pedagógica en las condiciones social concreta del Ecuador, como una alternativa de formación del docente universitario a corto y mediano plazo que se contextualiza en cursos, conferencias, seminarios y talleres dirigidos a potenciar la formación pedagógica en un nivel insipiente en las universidades ecuatorianas y en particular en la Facultad de Ciencia de la Educación e Idioma de la Universidad Estatal Península Santa Elena. En esta dirección se considera el diagnóstico de necesidades de los profesores en la dimensión pedagógica como punto de partida para la ubicación del claustro por niveles de desarrollo alcanzado en esta área del conocimiento.

De lo anterior, se deriva la importancia que se le concede a la formación pedagógica, pues contempla entre sus objetivos esenciales, la formación para un ejercicio responsable, ético y pertinente de la docencia, que se exprese en la autonomía y el compromiso con la calidad de su función educativa. Este objetivo se logra en la medida en que las vías que se utilicen para alcanzar tal fin se diseñen, ejecuten y evalúen, se expresen en indicadores relacionados con el desarrollo profesional como: interés profesional, satisfacción en el desempeño profesional, reflexión crítica en su actuación profesional, perseverancia, compro- 
miso con la calidad de la formación profesional, flexibilidad en la actuación profesional, dominio de conocimientos y habilidades profesionales.

Esta consideración explica la definición de las exigencias que caracterizan la formación pedagógica, ya que por su orientación debe dar respuesta a las necesidades del profesorado universitario, tener en cuenta los diversos contextos en que actúa el docente universitario, tener un carácter permanente, flexible y diferenciado por niveles de desarrollo para relacionar de manera acertada los intereses individuales e institucionales. Se necesita entonces que la pedagogía ponga, a través del docente, especial atención al desarrollo del hombre como individuo, para poder interactuar sobre él con acciones diferenciadas y, en ese sentido, el abordaje de la formación del docente de nivel superior debe centrarse en el estudio de las concepciones pedagógicas necesarias, analizando en su conjunto la profesión docente universitaria así como los procesos de institucionalización universitaria.

La formación pedagógica además, contribuye al desarrollo y consolidación de las funciones del profesor como guía del proceso docente educativo, a partir del análisis teórico y la dimensión práctica, elementos que sustentan su desarrollo profesional, como docente universitario y consolida la integración necesaria entre la docencia, la investigación y la vinculación con la práctica como principio básico del profesional que se forma en el aula universitaria, en el que el profesor juega un papel protagónico.

En el conocimiento de los fenómenos educacionales desempeña una función esencial el enfoque de sistema, que proporciona la orientación general para su estudio, como una realidad integral formada por componentes que cumplen determinadas funciones y mantienen formas estables de interacción entre ellos. Por esta razón, el conocimiento del enfoque de sistema presenta una importancia fundamental para la investigación educacional.

Un sistema no es un conglomerado de elementos yuxtapuestos mecánicamente, sino que representa leyes de totalidad, esto es, cualidades generales inherentes al conjunto, las cuales se diferencian de las características individuales de los componentes que lo integran. Es justamente la interacción entre los componentes del sistema lo que genera sus cualidades. Los fenómenos educacionales, al igual que todos los fenómenos sociales, están sujetos a leyes que los caracterizan como sistema. De aquí, la importancia que presenta el estudiar las cualidades generales de los sistemas para el dominio de la metodología de la investigación pedagógica. Estas cualidades son las siguientes: componentes, principio de jerarquía, estructura, y relaciones funcionales del sistema.

A criterio de los autores del presente reporte científico, un sistema de formación pedagógica dirigido a los docentes de nivel superior comprende todo el sistema de acciones conscientes, con carácter continuo, sistémico, personalizado, contextualizado a las necesidades y evaluable que propicia, a partir de los compromisos individuales y la experiencia teórico - práctica de los profesores universitarios, lograr la formación pedagógica necesaria para resolver las situaciones docentes que se presenten en el aula universitaria de manera que se pueda responder a las exigencias y expectativas de la educación terciaria en el siglo $\mathrm{XXI}$.

Los resultados obtenidos a través de las indagaciones teóricas y empíricas desarrolladas a lo largo del proceso investigativo, posibilitaron elaborar el sistema de formación pedagógica cuyos rasgos distintivos se revelan en la determinación de niveles, componentes y las relaciones entre ellos, atendiendo a la necesidad de que un sistema tenga una adecuada estructura, definidas sus propiedades y componentes o partes constituyentes entre los que pueden observarse los niveles que se declaran y definen en el presente informe.

El diseño de este sistema se fundamenta en la concepción teórica de la formación pedagógica que exige niveles para su realización. Cada una de estas vías de formación pedagógica contiene los elementos que de manera organizada y ordenada fundamentan la preparación de los profesores del claustro, teniendo en cuenta el objetivo, el objeto y el eje transversal del proceso de formación concreto, así como la realización del diagnóstico inicial de necesidades como punto de partida que conforman los componentes de la formación pedagógica.

Los autores consideran que el resultado de la formación pedagógica de los profesores es la práctica docente de los mismos y que requieren de ésta en conjugación con los intereses institucionales, lográndose derivar las etapas del sistema en acciones, que contribuyan a su preparación. En ese sentido se obtiene un sistema de formación pedagógica perfeccionado, fortalecido y desarrollador de conocimientos, habilidades, valores y modos de actuación del profesor universitario en correspondencia con las demandas sociales de la Facultad de Ciencia de la Educación e Idioma de la Universidad Estatal Península Santa Elena. 
La integración por tanto, de todos los componentes da lugar al sistema para lograr la formación pedagógica del profesor universitario, las etapas que conforman la lógica del proceso de formación, planteadas por Álvarez de Zayas (5), son asumidas por el autor para presentar la concepción de la formación pedagógica universitaria. Se utiliza como elemento esencial las etapas del proceso de formación, pero adecuadas al nivel de desarrollo de los profesores y considerando la evaluación-mejora como elemento permanente para perfeccionar el desempeño del profesor universitario.

Este sistema será aplicado de forma escalonada, por lo que su ejecución, desarrollo e implementación se programa para los años del 2015 al 2017. Esquemáticamente, el sistema de formación pedagógica del claustro puede representarse como se observa en la figura 1 a continuación.

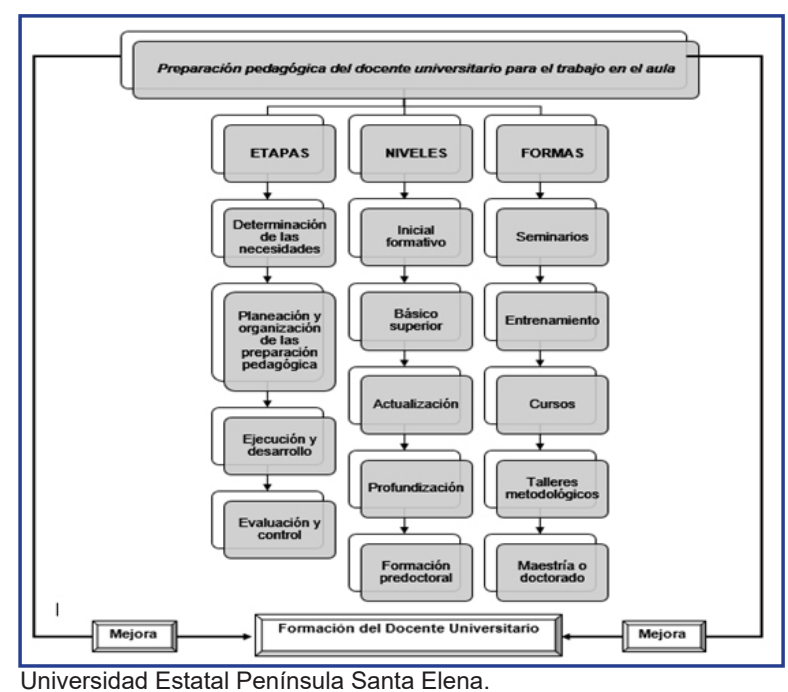

De la figura anterior puede observarse que existen relaciones de interdependencia e interacción entre los componentes del sistema, en este caso, entre las etapas, niveles y formas en que se implementará el sistema propuesto pudiendo observarse la existencia de relaciones de coordinación entre los tres componentes donde los niveles son el componente central que a su vez se subordinan los restantes dos componentes.

Atendiendo al análisis realizado se define como niveles de formación a los diferentes estadios de desarrollo de las habilidades docentes del claustro, desde el básico hasta el de formación pre-doctoral, que proporcionarán toda la formación e información, tanto de carácter básico y generalista como de mayor extensión y profundización en la teoría propia del conocimiento y la práctica pedagógica con carácter aplicado y creativo, que se determinan a partir de un sólido basamento científico, tecnológico, social y procedimental y que, al egresar de cada uno de ellos, el profesor participante del proceso de formación pedagógica lo haga habilitado para un desempeño profesional solvente que le permita afrontar las contingencias del ejercicio profesional docente y maneje las herramientas que posibiliten incorporar los conocimientos que vaya requiriendo para la solución de los problemas del aula universitaria.

A partir de ello se propone los siguientes niveles de formación y se determina la caracterización de cada uno de ellos para el caso del sistema de formación pedagógica del claustro de profesores de la Facultad de Ciencia de la Educación e Idioma de la Universidad Estatal Península Santa Elena.

Nivel inicial formativo: Incluye a todos los estudiantes que se encuentren cursando las carreras que se estudian en la Facultad de Ciencia de la Educación e Idioma de la Universidad Estatal Península Santa Elena, recibirán el Programa de la materia Pedagogía como asignatura obligatoria dentro del Currículo, con un número de horas 32. Persigue desarrollar las capacidades, disposiciones y actitudes de los profesores, con el fin de prepararles para acceder a la enseñanza y facilitarles la eficacia en su tarea docente. En el último año semestre o módulos optativos.

\section{Resultados esperados en este nivel de forma- ción pedagógica}

- Contribuir a la formación de valores morales, éticos y profesionales en los estudiantes, priorizando la honestidad y la solidaridad de gran importancia en la labor pedagógica.

- Desarrollar el pensamiento científico e investigativo que debe caracterizar a un profesional de las ciencias técnicas y humanísticas, promoviendo el espíritu investigativo, la superación y la creatividad en su actividad laboral y pensamiento crítico y autocritico.

- Desarrollar el liderazgo y la capacidad de trabajo en grupo.

Nivel básico superior. Incluye a aquellos docentes y directivos con jerarquía académica de tercer nivel en ramas del conocimiento diferentes a las Ciencias Pedagógicas que no acreditan formación pedagógica, pero poseen años de experiencia en la Educación Superior u otro nivel de enseñanza. Este nivel está relacionado con la fase de inducción relacionada en la literatura especializada y que se corresponde en el tiempo con los primeros años de ejercicio profesional, cuando los docentes universitarios aprenden en la práctica. Este nivel, incluye tres tipos de situaciones profe- 
sionales: profesores noveles que terminados sus estudios acceden por primera vez a la enseñanza universitaria como profesionales; profesores que regresan a la enseñanza después de varios años de distanciamiento del desempeño como docente y profesores que desempeñan nuevas tareas y necesitan de una formación específica.

\section{Resultados esperados en este nivel de formación pedagógica}

\begin{abstract}
- Contribuir al perfeccionamiento de la labor docente-educativa de los profesores mediante una formación en los aspectos pedagógicos fundamentales que caracterizan el proceso enseñanza-aprendizaje en la Educación Superior y en especial en el Ecuador.

- Contribuir al proceso de formación pedagógica de los profesores universitarios mediante la formación en los aspectos pedagógicos fundamentales que caracterizan el proceso docente-educativo en la Educación Superior, que permita mediante la reflexión teórica práctica de los mismos, aplicar los conocimientos, habilidades, valores y modos de actuación, adquiridos en los salones de las clases.
\end{abstract}

Nivel de actualización. Incluye a aquellos docentes y directivos, con tercer nivel terminado en Ciencias Pedagógicas y no acreditan cursos de actualización pedagógica en los últimos cinco años. Este nivel se relaciona con algunas etapas descritas en la literatura especializada y que se reconoce como momento o etapa de formación en ejercicio, formación continua, perfeccionamiento, actualización, reciclaje, desarrollo profesional y formación en servicio. Su principal característica es que incluye aquellas actividades que propician el desarrollo profesional del docente.

\section{Resultados esperados en este nivel de formación pedagógica}

- Contribuir a la formación permanente y profundización de los participantes en diferentes campos de la administración educativa enfocada a la dirección científica del proceso docente-educativo, que se desarrolla en las instituciones de educación superior.

- Analizar, con enfoque de sistema, el proceso docente educativo y las regularidades que se manifiestan en éste, valorando las funciones de planificación, organización, integración, dirección y control.

- Promover el estudio comparado de estas temáticas y dar a cada participante la oportunidad de evaluar aspectos importantes de la dirección del proceso docente-educativo, de especial interés para la Universidad Estatal
Península Santa Elena.

Nivel de profundización. Incluye a aquellos docentes y directivos que acreditan cursos de actualización pedagógica dentro de los últimos cinco años. Estos deben integrarse directamente a los programas de maestría, relacionados con las Ciencias Pedagógicas y Ciencias de la Educación.

\section{Resultados esperados en este nivel de formación pedagógica}

- Desarrollar capacidades en los profesores para la gestión y la innovación en instituciones educativas como parte de su formación pedagógica.

Nivel de formación pre-doctoral. Incluye a aquellos docentes y directivos que han transitado por los diferentes niveles del sistema propuesto.

\section{Resultados esperados en este nivel de formación pedagógica}

- Profundizar en los fundamentos teóricos y metodológicos que sustentan el proceso de investigación en educación desde los diferentes paradigmas que lo soportan y la utilización práctica de métodos y técnicas científicas.

- Desarrollar habilidades de búsqueda, selección y evaluación de la información para la construcción de un marco teórico referencial de la investigación durante el proceso de formación doctoral y la producción y comunicación de artículos científicos.

\section{Evidencias del desarrollo del Sistema de formación pedagógica de los profesores}

Desde el inicio de la ejecución del sistema para la formación pedagógica del claustro se han realizado un ciclo de Conferencias, Seminarios, Talleres, Cursos y se profundizó en el trabajo didáctico de las carreras. En ello han participado profesores de las carreras y los directivos académicos, en función de mejorar la formación pedagógica del claustro y la transformación de los indicadores, que permiten observar el comportamiento del acceso y la retención del estudiantado en las cohortes de la Facultad de Ciencias de la Educación e Idiomas, de la Universidad Estatal Península Santa Elena.

Entre las principales estrategias y acciones a desarrollar en los años 2015-2017, por parte del equipo de asesores, liderados por los autores del presente trabajo, se encuentran las que se rela- 
cionan a continuación:

- Asesoramiento didáctico a directores de carreras.

- Actividades metodológicas sobre los lineamientos de la evaluación del proceso de evaluación de los aprendizajes en los estudiantes.

- Talleres para la confección de materiales de estudio para la preparación de los estudiantes en competencias generales. (Guías de Estudio, Base Orientadora para la Acción. BOA).

- Preparación didáctica del equipo de docentes para enfrentar el proceso de capacitación a estudiantes de todas las carreras, en competencias generales.

- Preparación didáctica del equipo de docentes para la confección, aplicación y revisión de examen de competencias generales.

- Revisión de las líneas de investigación de la Universidad y un taller metodológico sobre la vinculación, docencia e investigación, con la comunidad.

- Curso de tutores, asesores y lectores (oponentes) de tesis. El producto final fue la revisión y reelaboración de guía metodológica para la realización de trabajos de titulación en la Facultad de Ciencias de la Educación e Idiomas.

- Asesoramiento metodológico para la preparación de la planificación docente en la Universidad.

- Curso sobre marco lógico para la gestión de proyectos.

- Talleres de revisión y análisis crítico de las mallas curriculares de todas las carreras. Presentación del informe final.

- Confección de propuesta de reglamento de evaluación del desempeño docente.

- Confección de modelo por competencias para la Universidad.

- Curso taller de modelo por competencias para la confección de los pensum de las carreras. Confección de los planes de estudio de todas las carreras.

- Asesoría para elaboración de programas analíticos (Sílabos) de las asignaturas. Completamiento de los programas analíticos (Sílabos) de las asignaturas a impartir.

- Elaboración de procedimiento para realización de examen de conocimientos relevantes y traslados de carrera.

- Revisión y perfeccionamiento del proyecto del curso de nivelación para la admisión de estudiantes en la Facultad de Ciencias de la Educación e Idiomas, de la Universidad Estatal Península Santa Elena.

Todo lo anterior, acredita la intensidad que ha alcanzado la actividad académica y permite hacer juicios de valor con respecto a la transformación de la actividad académica y la implicación de los docentes, lo que demuestra los avances, en materia de formación pedagógica y de mejora continua, en la calidad del proceso de enseñanza y aprendizaje, en la Facultad de Ciencias de la Educación e Idioma de la Universidad Estatal Península Santa Elena.

\section{Control y seguimiento a la forma- ción pedagógica del claustro de la Facultad}

La dirección efectiva del proceso de formación pedagógica de los profesores universitario, requiere del conocimiento de cómo se realiza el aprendizaje de los profesores que participan en los cursos propuestos y cuáles son sus resultados, dado que la evaluación del aprendizaje es una parte esencial del proceso de enseñanza, que permite el control y seguimiento de la asimilación de los conocimientos, habilidades y hábitos que los profesores adquieren como resultado del proceso.

Permite comprobar el grado en que se cumplen los objetivos propuestos y constituye un elemento de retroalimentación y dirección del proceso de formación pedagógica. Consideramos el control y el seguimiento como un proceso que permite:

- Conocer -en primer lugar- a los profesores, como destinatarios y sujetos activos de su aprendizaje, sus necesidades, intereses, expectativas y su experiencia anterior, incluyendo sus aciertos y errores.

- En segundo lugar, el proceso de enseñanza-aprendizaje que comprende los propósitos, los medios y los elementos que favorecen o dificultan dicho aprendizaje.

- En tercer lugar, el conocimiento de los resultados del proceso de formación pedagógica, entendidos como cambios relevantes y significativos en la experiencia y en las capacidades de los profesores.

- Valorar todos y cada uno de los elementos que constituyen la actividad educativa, desde su planificación y programación en los distintos niveles, hasta los resultados, pasando por la aplicación y puesta en práctica de las estrategias y actividades previstas. En esta valoración interesa destacar los criterios cualitativos y la determinación de las necesidades de mejora.

- Decidir acerca de las medidas de ayuda individualizada necesarias para lograr el desarrollo óptimo en cada profesor, en función de sus posibilidades.

Este ámbito también comprende las decisiones 
sobre posibles y necesarios cambios en la programación y en la práctica docente, en la metodología y en los recursos, así como sobre las calificaciones, promoción y titulación correspondientes.

El control y seguimiento de la formación pedagógica se realiza sistemáticamente manteniendo un monitoreo por parte de los niveles de dirección de la institución, visitas a clases sin previa comunicación a docentes por las diferentes instancias de dirección, entrevistas formales e informales a estudiantes y profesores, encuestas dirigidas a conocer el grado de satisfacción de profesores y estudiantes de la implementación referentes al proceso de formación pedagógica (profesores) y respecto a la calidad de las clases (estudiantes).

Por otra parte, el control de la ejecución del Sistema propuesto se lleva a cabo teniendo en cuenta también los indicadores que establecen los órganos de control de la Educación Superior en el Ecuador. Es por ello, que se ajustan con frecuencia las estrategias seguidas y en tal sentido la propuesta es flexible y garantiza la retroalimentación y la adecuación inmediata a las necesidades y demandas del entorno.

\section{Resultados obtenidos en la concepción, validación y ejecución del sistema de formación pedagógica}

Los resultados obtenidos, a partir del empleo de métodos y técnicas tales como: método de criterios de expertos (Método Delphi) por la posibilidad que ofrece de obtener información de forma independiente, de intercambio de información y de evitar evaluaciones superficiales; Técnica de ladov para conocer el grado de satisfacción de los profesores que participaron en el proceso de formación pedagógica , se realiza una medición de los cursos, conferencias, seminarios y talleres planificados por niveles de formación después de haber recibidos los contenidos de los cursos impartidos, esto permitió contrastar el grado de satisfacción de los profesores y constituye una de las vías utilizadas en la implementación del sistema.

Este Método Delphi permitió extraer la información de los expertos que conforman un grupo heterogéneo, analizar las convergencias de opiniones en torno a la propuesta que se aborda en la investigación, facilitar a los expertos entrevistados emitir sus opiniones sin saber qué es lo que otros colegas opinan, llegando a un consenso de ideas, reflexiones, criterios incidiendo en la mejora de la problemática planteada. Se basó en la utilización sistemática de juicios de opiniones de un grupo de expertos hasta llegar a un acuerdo, en este proceso se trató de evitar las influencias de individuos o grupos dominantes y al mismo tiempo existió la retroalimentación facilitando el acuerdo final.

Los pasos lógicos seguidos para la aplicación del Método Delphi fueron: la concepción inicial del sistema propuesto, se realiza una caracterización de la preparación pedagógica del claustro de profesores haciendo énfasis en el campo de acción que será contextualizado el mismo, se describen las vías que lo conforman, así como la fundamentación pedagógica teniendo en cuenta los fundamentos filosóficos, psicopedagógicos y didácticos en que se sustenta el sistema, se explicitan los estándares tenidos en cuenta en su elaboración para el control y seguimiento, así como una de las vías utilizadas para la implementación del mismo en la Facultad de Ciencia de la Educación e Idioma de la Universidad Estatal Península Santa Elena.

Los expertos, por unanimidad indicaron que lo dejaran en el anonimato, pero se convino la posibilidad de informar su procedencia y sus características distintivas, de los 12 expertos seleccionados, 5 son de nacionalidad cubana y poseen el grado científico de Doctores en Ciencias y con una experiencia docente de más de 20 años en la educación superior, 3 son de nacionalidad ecuatoriana y poseen grado científico de Doctores en Ciencias y con una experiencia docente de más de 20 años en la educación superior, 3 son de nacionalidad española y poseen el grado científico de Doctores en Ciencias y con una experiencia docente de más de 20 años en la educación superior, 1 es de nacionalidad Estados Unidos y poseen el grado científico de Doctores en Ciencias y con una experiencia docente de más de 20 años en la educación superior.

Los 6 criterios emitidos por los expertos, después de realizar las rondas correspondientes atendiendo a la metodología establecida se ponderaron y validaron:

Criterio 1: Los expertos consideran que el Sistema que se propone es muy pertinente, novedoso y aplicable en el contexto ecuatoriano.

Criterio 2: Los expertos consideran que en el diagnóstico realizado debió ampliarse el muestreo a todas las Universidades posicionadas legalmente en este territorio.

Criterio 3: Consideran los expertos que debe incluirse en un anexo lo relacionado con el Reglamento de Admisión y Nivelación para el acceso de los estudiantes a la Facultad de Ciencias de 
la Educación e Idiomas de la Universidad Estatal Península Santa Elena.

Criterio 4: Consideran los expertos que se deben mencionar las formas de control, seguimiento y evaluación, empleadas en la implementación del Sistema.

Criterio 5: Existe un consenso en los 12 expertos que participan en el proceso de validación, que el Sistema propuesto puede ser aplicado ya que es muy pertinente, importante y novedoso para todas las Universidades del Ecuador.

Criterio 6: Los 9 expertos extranjeros (Cuba (5), España (3) y EEUU (1)) que participan en el proceso se pronuncian y establecen comparaciones con los existentes en sus países resaltando la percepción, concepción y originalidad en las vías utilizadas para la implementación del Sistema.

De los resultados obtenidos al aplicar el paquete estadístico orientado al ámbito de las Ciencias Sociales, SPSS para Windows versión 15.0, se infiere que el comportamiento del coeficiente de variación, valor de las ligaduras y el coeficiente Kendall o coeficiente de correlación de rango, demuestran el alto grado de concordancia de los expertos con los criterios emitidos que validan.

\section{Conclusiones}

En la presente investigación se explicitaron los fundamentos teóricos metodológicos que sustentan la formación pedagógica de los docentes, mediante el análisis y contextualización de las concepciones teóricas asumidas para sustentar el sistema de cursos, conferencias, seminarios y talleres dirigidos a la formación pedagógica del claustro de profesores de la Facultad de Ciencias de la Educación e Idiomas.

Se realizó un diagnóstico, determinándose las regularidades con respecto a los niveles de formación pedagógica de los profesores que conforman el claustro y permitió establecer la formación pedagógica, para contribuir a la formación de los profesores de la Facultad.

Se valida el sistema de cursos, conferencias, seminarios y talleres, dirigido a lograr la formación pedagógica del claustro, aplicando métodos cualitativos y cuantitativos, así como técnicas científicas que garantizan los resultados obtenidos durante el proceso de investigación.

Se muestran resultados que validan la pertinencia y el impacto que ha tenido en la formación del claustro de la Facultad.
Se recomienda generalizar estos resultados a las demás Facultades de la Universidad Estatal Península de Santa Elena.

\section{Referencias bibliográficas}

1.García, L. Autoperfeccionamiento docente y creatividad, 1ra Edición, Pueblo y Educación, 185, La Habana, Cuba (1996).

2.Rosenshine, B., Meister, C., \& Chapman, S. Teaching students to generate questions: A review of the intervention studies. Review of Educational Research, 66(2), 181-221 (1996).

3.Núnez Rojas, Nemecio y Palacios Contreras, Pedro Gonzalo. Modelo teórico para un programa decapacitación continua de docentes en servicio en educación básica. Tesis presentada en opción algrado científico de doctor en ciencias de la educación. Lambayeque. Perú. (2002).

4. Addine Fernández, F. La didáctica general y su enseñanza en la Educación Superior. Pedagógica. Aportes e impacto. Compilación de los principales resultados investigativos en opción al grado científico de Doctor en Ciencias. La Habana. Cuba.(2010).

5. Álvarez De Zayas, Carlos M. La Pedagogía como ciencia. Epistemología de la Educación, 1 ra Edición, Pueblo y Educación, 1-254, La Habana, Cuba (1999). 